\title{
P03-6-23 Poster session
}

\section{Study for chemical composition and wound repair activity of Bletilla formosana polysaccharide}

\author{
Jing-Jy Cheng, Mei-Kuang Lu, Chi-Hsein Chao \\ National Research Institute of Chinese Medicine, Taiwan
}

Wound repair is highly complex, comprising a series of coordinated and overlapping processes. The management of a chronic wound has become a major therapeutic challenge, and it is a problem that will only escalate with the increasing incidence of conditions that impede wound healing, such as diabetes, obesity and vascular disorders. Bletilla formosana (B. formosana, is used as common bletilla tuber, which belongs to one kind of the Taiwan peculiar plants. Polysaccharide is considered important with many biological functions. Therefore, in this study, we first propagate the culture of $B$. formosana for 45 days, isolation of its polysaccharide (BFP) and to identify the sugar compositions and MW distribution. Moderate IL- $\beta$ and TNF- $\alpha$ secreation was found after treatment with BFP for $24 \mathrm{hrs}$ in macrophages. BFP also showed significant potency on EC migration and also increased angiogenesis in vitro. VE-cadherin, $\beta$-catenin and Wnt1 expression were increased while E-cadherin expression decreased after BFP incubation on ECs. The moderate inflammatory effect, enhancement of EC migration and alternation of Wnt/ $\beta$-catenin pathway might correlate with the wound-healing activity of BFP. Working on the study and development of Taiwan peculiar plants is valuable especially using tissue culture is a convenient way to propagate plants fast and efficient. As chronic wound is a problem that will escalate with the increasing incidence of conditions that impede wound healing, such as diabetes, obesity and vascular disorders, we anticipate our results will further provide evidence of BFP as a new therapeutic reference for wound repair. 